\title{
The UMIST database for astrochemistry $1999^{\star}$
}

\author{
Y.H. Le Teuff, T.J. Millar, and A.J. Markwick \\ Department of Physics, UMIST, PO Box 88, Manchester M60 1QD, UK
}

Received January 31; accepted July 4, 2000

\begin{abstract}
We report a new version of the UMIST database for astrochemistry. The previous (1995) version has been updated and its format has been revised. The database contains the rate coefficients, temperature ranges and - where available - the temperature dependence of 4113 gas-phase reactions important in astrophysical environments. The data involve 396 species and 12 elements. We have also tabulated permanent electric dipole moments of the neutral species and heats of formation. A new table lists the photo process cross sections (ionisation, dissociation, fragmentation) for a few species for which these quantities have been measured. Data for Deuterium fractionation are given in a separate table. Finally, a new online Java applet for data extraction has been created and its use is explained in detail. The detailed new datafiles and associated software are available on the World Wide Web at http://www.rate99.co.uk.
\end{abstract}

Key words: molecular data - molecular processes - ISM: molecules - circumstellar matter

\section{Introduction}

The UMIST Database is widely used by the scientific community in modelling chemical kinetics in the interstellar medium. In this paper, we report on the latest release of the database and on electronic access to our full range of data and related codes. In recent years, some very large models, often containing up to 10000 reactions, have been published. Our latest release takes a much more circumspect approach with the number of reactions extended by 249 compared to the previous release (Millar et al. 1997), to a total of 4113. Large systems often take a generic approach to the rate coefficients and chemical

Send offprint requests to: Y.H. Le Teuff

* Table 8 is only available in electronic form at

http://www.edpsciences.org and http://www.rate99.co.uk reactions included by extending particular reactions applicable to smaller species to much larger systems. While this is a useful approach in determining whether or not large molecules may form, it is not based generally on thermodynamics which can affect particular reactions.

In recent years, there has been an increasing need to model the chemical kinetics of hot circumstellar envelopes and interstellar shocks, with the result that we have reviewed the temperature dependence and temperature range validity of each reaction. Although not yet totally accurate, we believe this new piece of data should make models noticeably more reliable. In addition, we have included a number of three-body reactions which are of particular importance in high density environments, such as protoplanetary disks. In Sect. 2, the new conventions adopted to tabulate the species and their possible isomers are explained. Also, a few words of caution are given on the data, mainly concerning the lack of distinction between certain isomers. Section 3 describes the content of the database and the new format used. Section 4 gives details on the new data that have been included in our online database set. Finally, Sect. 5 reports on the use of the newly developed Java applet which has been designed to enable the user to select and save to a local disk particular data.

\section{The species and related data}

The whole set of reactions contains 396 species (excluding the electrons), made from the 12 following elements: $\mathrm{H}$, $\mathrm{He}, \mathrm{C}, \mathrm{N}, \mathrm{O}, \mathrm{Na}, \mathrm{Mg}, \mathrm{Si}, \mathrm{P}, \mathrm{S}, \mathrm{Cl}, \mathrm{Fe}$. The species are tabulated in Table 1 and their heats of formation, where available, are listed in Table 2, while the dipole moments of the neutral molecules are given in Table 3 .

\subsection{The nomenclature}

The functional site on the species has been highlighted whenever possible by writing it under a pseudo-developed 
form on the right of the species formula. Carboxylic acids end in "COOH", aldehydes in "CHO", alcohols in "COH", thiols in "SH", etc. For unsaturated or radical compounds the situation is more complex. For cyanopolyynes $\mathrm{HC}_{n} \mathrm{~N}$, a condensed formula is sufficient to describe the molecule because its triple and single bonds are regularly alternated. For other species, the formula should appear obvious: the charge on $\mathrm{C} 2 \mathrm{H} 70+$ is not on the same atom as for $\mathrm{C} 2 \mathrm{H} 6 \mathrm{OH}+$; $\mathrm{HCN}$ carries $\mathrm{H}$ on $\mathrm{C}$, whereas $\mathrm{HNC}$ carries $\mathrm{H}$ on the $\mathrm{N}$ atom. All the $\mathrm{C}_{n} \mathrm{H}_{m}$ molecules are meant to be linear chains when written under their condensed form.

But there are difficult cases: for example, C3H3+ and $\mathrm{H} 2 \mathrm{C} 3 \mathrm{H}+$ differ by their geometry: $\mathrm{C} 3 \mathrm{H} 3+$ is a cycle, whereas $\mathrm{H} 2 \mathrm{C} 3 \mathrm{H}+$ is linear with one triple bond $\left(\mathrm{H}_{2} \mathrm{C}-\mathrm{C} \equiv \mathrm{CH}^{+}\right)$; Also, due to the lack of precision on the isomeric nature of $\mathrm{C}_{3} \mathrm{H}_{n}, n=0 . .3$, used in experiments and mentioned in the literature, no distinction has been made between l- $\mathrm{C}_{3} \mathrm{H}_{n}$ and $\mathrm{c}-\mathrm{C}_{3} \mathrm{H}_{n}$, as sometimes experiments cannot discriminate between the isomers (e.g., propyne $\mathrm{HC} \equiv \mathrm{C}-\mathrm{CH}_{3}$ and allene $\mathrm{H}_{2} \mathrm{C}=\mathrm{C}=\mathrm{CH}_{2}$ ). Therefore for more detailed data, the user is encouraged to refer to the bibliographic references.

The other delicate isomer distinction is between H2CCC, which carries both $\mathrm{H}$ on the same carbon atom, and $\mathrm{C} 3 \mathrm{H} 2$, where both $\mathrm{H}$ are on distinct $\mathrm{C}$ atoms.

\subsection{The conventions}

Termolecular and collider reactions contain a catalyst species, the nature of which is not specified and is simply noted "M". Software - available on-line on http://www.rate99.co.uk - which turns kinetic reaction sets into differential equation subroutines, automatically replaces $\mathrm{M}$ by the medium number density. To increase data readability for the user, the names of the two-letter elements have been rewritten as a capital letter followed by a lower-case letter (e.g. $\mathrm{HE} \rightarrow \mathrm{He}$; SI $\rightarrow \mathrm{Si}$ ) and the electrons have been changed from "ELECTR" to "e-".

\section{The reaction set}

Table 8 contains all the reactions and associated rate coefficients, and is available online at http://www.rate99.co.uk http://www.edpsciences.org

\subsection{The new entry format}

The number of observed and predicted astrophysical species increases steadily with time, and with it, the size of their formula (e.g. ethyl methyl ether, $\mathrm{C}_{2} \mathrm{H}_{5} \mathrm{OCH}_{3}$, has been discussed as a possible interstellar molecule). To account for this, we have altered the format for the names of the species by increasing by one letter, making them 8-character strings. Also, the smallest products in a four-product reaction are mostly $\mathrm{H}$ and/or He, therefore only 4 characters have been allocated for the last two products. The necessity to consider termolecular reactions for high density environments means that a third species must be included on the reactant side of these reactions. To account for that, each reaction now comprises three reactants and four products. Our new reaction format reads:

I, R1, R2, R3, P1, P2, P3, P4, $\alpha, \beta, \gamma$, flags

where $\mathrm{I}$ is the reaction number, $\mathrm{R} 1$ to $\mathrm{R} 3$ are the reactants, $\mathrm{P} 1$ to $\mathrm{P} 4$ are the products, and $\alpha, \beta$ and $\gamma$ are the constants used to determine the rate coefficients. The series of flags is a string of 16 characters and/or digits that store respectively:

- the kind of data: measured M, estimated E, calculated $\mathrm{C}$ or literature search L, with format A1. Here "literature search" means that the given datum is a compilation of several other data (measured and/or calculated). The sources of these data are mainly Baulch et al. (1992) and the NIST database (Mallard et al. 1998);

- the lowest and highest temperatures defining the temperature range, format 2(I5). Each temperature is given as an integer number of kelvins in the range $10<T<41000 \mathrm{~K}$

- the error on the rate value, format A1. The following scheme has been used:

- "A". Error $<25 \%$

- "B". Error < 50\%

- "C". Error within a factor of 2

- "D". Error within an order of magnitude

- "E". Highly uncertain;

- the reference code, format A4. The references are listed in Table 4.

The full entry format in Fortran is correspondingly written as:

I4, 5(1X, A8), 2(1X, A4), 1X, 1PE8.2, 3X, OPF5.2, 2X, OPF8.1, A1, 2(I5), A1, A4.

\subsection{Calculation of the rates from $\alpha, \beta$ and $\gamma$}

For two- or three-body reactions, the rate coefficient is given by:

$k=\alpha(T / 300)^{\beta} \exp (-\gamma / T) \quad \mathrm{cm}^{3} \mathrm{~s}^{-1}$

where $T$ is the gas temperature.

For direct cosmic-ray ionisation $(\mathrm{R} 2=\mathrm{CRP})$ :

$k=\alpha \quad \mathrm{s}^{-1}$

whereas for cosmic-ray-induced photoreactions $(\mathrm{R} 2=$ CRPHOT):

$k=\alpha(T / 300)^{\beta} \gamma /(1-\omega) \quad \mathrm{s}^{-1}$ 
Table 1. List of the species found in the database. The 396 species are classified by their number of atoms and are vertically ordered by mass

\begin{tabular}{|c|c|c|c|c|c|c|c|c|}
\hline 1 & 2 & 3 & 4 & Nun & $\begin{array}{l}\text { er of atoms } \\
6\end{array}$ & 7 & 8 & $\geq 9$ \\
\hline $\begin{array}{l}\mathrm{H}- \\
\mathrm{H}+ \\
\mathrm{H} \\
\mathrm{He}+ \\
\mathrm{He} \\
\mathrm{C}- \\
\mathrm{C}+ \\
\mathrm{C} \\
\mathrm{N}+ \\
\mathrm{N} \\
\mathrm{O}- \\
\mathrm{O}+ \\
\mathrm{O} \\
\mathrm{Na}+ \\
\mathrm{Na} \\
\mathrm{Mg}+ \\
\mathrm{Mg} \\
\mathrm{Si}+ \\
\mathrm{Si} \\
\mathrm{P}+ \\
\mathrm{P} \\
\mathrm{S}- \\
\mathrm{S}+ \\
\mathrm{S} \\
\mathrm{Cl}+ \\
\mathrm{Cl} \\
\mathrm{Fe}+ \\
\mathrm{Fe}\end{array}$ & $\begin{array}{l}\mathrm{H} 2+ \\
\mathrm{H} 2 \\
\mathrm{HeH}+ \\
\mathrm{CH}+ \\
\mathrm{CH} \\
\mathrm{NH}+ \\
\mathrm{NH} \\
\mathrm{OH}+ \\
\mathrm{OH}- \\
\mathrm{OH} \\
\mathrm{C} 2+ \\
\mathrm{C} 2 \\
\mathrm{CN}+ \\
\mathrm{CN}- \\
\mathrm{CN} \\
\mathrm{CO}+ \\
\mathrm{CO} \\
\mathrm{N} 2+ \\
\mathrm{N} 2 \\
\mathrm{SiH}+ \\
\mathrm{SiH} \\
\mathrm{NO}+ \\
\mathrm{NO} \\
\mathrm{PH}+ \\
\mathrm{PH} \\
\mathrm{O} 2+ \\
\mathrm{O} 2 \\
\mathrm{HS}+ \\
\mathrm{HS} \\
\mathrm{HCl} \\
\mathrm{HCl}+ \\
\mathrm{SiC}+ \\
\mathrm{SiC} \\
\mathrm{SiN}+ \\
\mathrm{SiN} \\
\mathrm{CP}+ \\
\mathrm{CP} \\
\mathrm{CS}+ \\
\mathrm{CS} \\
\mathrm{SiS}+ \\
\mathrm{SiO}+ \\
\mathrm{SiO} \\
\mathrm{PN}+ \\
\mathrm{PN} \\
\mathrm{NS}+ \\
\mathrm{NS} \\
\mathrm{PO}+ \\
\mathrm{PO} \\
\mathrm{CCl}+ \\
\mathrm{CCl} \\
\mathrm{SO}+ \\
\mathrm{SO} \\
\mathrm{ClO}+ \\
\end{array}$ & 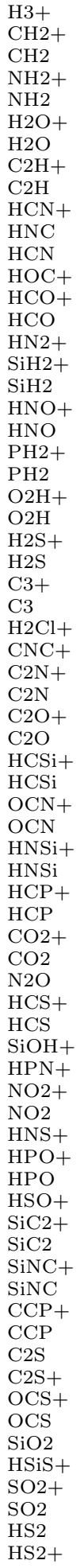 & 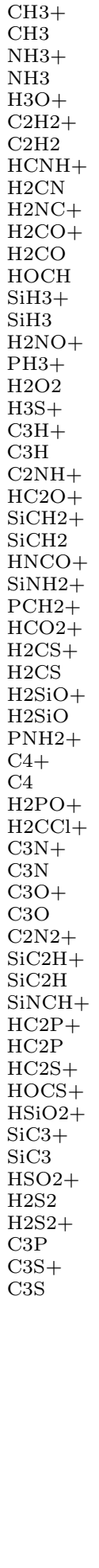 & $\begin{array}{l}\text { CH4+ } \\
\text { CH4 } \\
\text { NH4+ } \\
\text { C2H3+ } \\
\text { C2H3 } \\
\text { CH2NH } \\
\text { H3CO+ } \\
\text { SiH4+ } \\
\text { SiH4 } \\
\text { C3H2+ } \\
\text { C3H2 } \\
\text { H2CCC } \\
\text { CH2CN+ } \\
\text { CH2CN } \\
\text { CH2CO+ } \\
\text { CH2CO } \\
\text { SiCH3+ } \\
\text { SiCH3 } \\
\text { CH2PH } \\
\text { PCH3+ } \\
\text { HCOOH } \\
\text { H3CS+ } \\
\text { H3SiO+ } \\
\text { PNH3+ } \\
\text { C4H+ } \\
\text { C4H } \\
\text { HC3N+ } \\
\text { HC3N } \\
\text { HC3O+ } \\
\text { SiC2H2+ } \\
\text { SiC2H2 } \\
\text { PC2H2+ } \\
\text { C5+ } \\
\text { C5 } \\
\text { C4N+ } \\
\text { SiC3H+ }+ \\
\text { SiC3H } \\
\text { H3S2+ } \\
\text { PC3H+ } \\
\text { HC3S+ } \\
\text { SiC4+ } \\
\text { SiC4 } \\
\text { C4P+ } \\
\text { C4P } \\
\text { C4S+ }+ \\
\text { C4S }\end{array}$ & $\begin{array}{l}\mathrm{CH} 5+ \\
\mathrm{C} 2 \mathrm{H} 4+ \\
\mathrm{C} 2 \mathrm{H} 4 \\
\mathrm{CH} 2 \mathrm{NH} 2+ \\
\mathrm{CH} 4 \mathrm{~N}+ \\
\mathrm{CH} 3 \mathrm{OH}+ \\
\mathrm{CH} 3 \mathrm{OH} \\
\mathrm{SiH} 5+ \\
\mathrm{C} 3 \mathrm{H} 3+ \\
\mathrm{H} 2 \mathrm{C} 3 \mathrm{H}+ \\
\mathrm{C} 3 \mathrm{H} 3 \\
\mathrm{CH} 3 \mathrm{CN} \\
\mathrm{CH} 3 \mathrm{CN}+ \\
\mathrm{CH} 3 \mathrm{CO}+ \\
\mathrm{SiCH} 4+ \\
\mathrm{PCH} 4+ \\
\mathrm{HCOOH} 2+ \\
\mathrm{C} 4 \mathrm{H} 2+ \\
\mathrm{C} 4 \mathrm{H} 2 \\
\mathrm{H} 2 \mathrm{C} 3 \mathrm{~N}+ \\
\mathrm{C} 3 \mathrm{H} 2 \mathrm{O}+ \\
\text { SiC2H3+ } \\
\text { PC2H3+ } \\
\mathrm{C} 5 \mathrm{H}+ \\
\text { C5H } \\
\mathrm{HC} 4 \mathrm{~N}+ \\
\text { SiC3H2+ } \\
\text { C6+ } \\
\text { C6 } \\
\text { C5N+ } \\
\mathrm{C} 5 \mathrm{~N} \\
\text { SiC4H+ } \\
\text { PC4H+ } \\
\mathrm{HC} 4 \mathrm{~S}+\end{array}$ & $\begin{array}{l}\mathrm{C} 2 \mathrm{H} 5+ \\
\text { C2H5 } \\
\text { CH3OH2+ } \\
\text { C3H4+ } \\
\text { C3H4 } \\
\text { H4C2N+ } \\
\text { CH3CHO+ } \\
\text { CH3CHO } \\
\text { C4H3+ } \\
\text { C4H3 } \\
\text { H3C3N } \\
\text { H3C3O+ } \\
\text { PC2H4+ } \\
\text { C5H2+ } \\
\text { C5H2 } \\
\text { H2C4N+ } \\
\text { C6H+ } \\
\text { C6H } \\
\text { HC5N+ }+ \\
\text { HC5N } \\
\text { C7+ } \\
\text { C7 }\end{array}$ & $\begin{array}{l}\mathrm{C} 2 \mathrm{H} 6+ \\
\mathrm{C} 2 \mathrm{H} 6 \\
\mathrm{C} 3 \mathrm{H} 5+ \\
\mathrm{H} 5 \mathrm{C} 2 \mathrm{O}+ \\
\mathrm{C} 4 \mathrm{H} 4+ \\
\mathrm{H} 4 \mathrm{C} 3 \mathrm{~N}+ \\
\text { COOCH4+ } \\
\text { HCOOCH3 } \\
\text { C5H3+ } \\
\mathrm{H} 3 \mathrm{C} 4 \mathrm{~N}+ \\
\mathrm{H} 3 \mathrm{C} 4 \mathrm{~N} \\
\mathrm{C} 6 \mathrm{H} 2+ \\
\mathrm{C} 6 \mathrm{H} 2 \\
\mathrm{H} 2 \mathrm{C} 5 \mathrm{~N}+ \\
\text { C7H+ } \\
\text { C7H } \\
\text { C8+ } \\
\text { C8 } \\
\text { C7N+ } \\
\text { C7N }\end{array}$ & 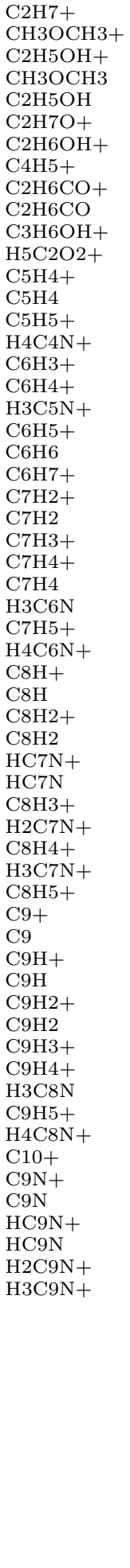 \\
\hline
\end{tabular}


Table 2. Species and heats of formation in $\mathrm{kJ} \mathrm{mol}^{-1}$ at $0 \mathrm{~K}$

\begin{tabular}{|c|c|c|c|c|c|c|c|c|c|}
\hline $\mathrm{H}$ & 216.0 & $\mathrm{He}$ & 0.0 & $\mathrm{C}$ & 711.2 & $\mathrm{~N}$ & 470.8 & $\mathrm{O}$ & 246.8 \\
\hline $\mathrm{Na}$ & 107.6 & $\mathrm{Mg}$ & 146.5 & $\mathrm{Si}$ & 446.0 & $\mathrm{P}$ & 315.6 & $\mathrm{~S}$ & 274.7 \\
\hline $\mathrm{Cl}$ & 119.6 & $\mathrm{Fe}$ & 414.0 & $\mathrm{H} 2$ & 0.0 & $\mathrm{CH}$ & 592.5 & $\mathrm{NH}$ & 376.5 \\
\hline $\mathrm{OH}$ & 38.4 & $\mathrm{SiH}$ & 374.9 & $\mathrm{PH}$ & 237.0 & HS & 136.5 & $\mathrm{HCl}$ & -92.1 \\
\hline $\mathrm{C} 2$ & 817.0 & $\mathrm{CN}$ & 436.8 & $\mathrm{CO}$ & -113.8 & $\mathrm{SiC}$ & 714.7 & $\mathrm{CP}$ & 447.6 \\
\hline $\mathrm{CS}$ & 277.1 & $\mathrm{CCl}$ & 384.0 & $\mathrm{~N} 2$ & 0.0 & $\mathrm{NO}$ & 89.8 & $\mathrm{SiN}$ & 371.2 \\
\hline $\mathrm{PN}$ & 105.8 & NS & 263.0 & $\mathrm{O} 2$ & 0.0 & $\mathrm{SiO}$ & -101.6 & $\mathrm{PO}$ & -32.0 \\
\hline $\mathrm{SO}$ & 5.0 & $\mathrm{ClO}$ & 101.8 & $\mathrm{SiS}$ & 104.6 & $\mathrm{~S} 2$ & 128.3 & $\mathrm{CH} 2$ & 390.0 \\
\hline NH2 & 191.6 & $\mathrm{H} 2 \mathrm{O}$ & -238.9 & $\mathrm{SiH} 2$ & 289.0 & PH2 & 112.3 & $\mathrm{H} 2 \mathrm{~S}$ & -17.6 \\
\hline $\mathrm{C} 2 \mathrm{H}$ & 560.0 & $\mathrm{HCN}$ & 135.5 & $\mathrm{HNC}$ & 201.0 & $\mathrm{HCO}$ & 44.8 & $\mathrm{HCSi}$ & $\ldots \ldots$ \\
\hline $\mathrm{HCP}$ & 167.0 & HCS & 310.0 & $\mathrm{HNO}$ & 100.0 & HNSi & 162.8 & $\mathrm{O} 2 \mathrm{H}$ & 13.4 \\
\hline HPO & $\ldots \ldots$ & HS2 & $\ldots \ldots$ & C3 & 831.0 & $\mathrm{CCN}$ & 556.0 & $\mathrm{CCO}$ & 282.6 \\
\hline $\mathrm{SiC} 2$ & 610.0 & $\mathrm{CCP}$ & $\ldots \ldots$ & $\mathrm{C} 2 \mathrm{~S}$ & 586.0 & $\mathrm{OCN}$ & 154.0 & $\mathrm{SiNC}$ & $\ldots \ldots$ \\
\hline $\mathrm{CO} 2$ & -393.1 & OCS & -142.0 & $\mathrm{~N} 2 \mathrm{O}$ & 85.5 & $\mathrm{NO} 2$ & 36.0 & $\mathrm{SiO} 2$ & -281.2 \\
\hline $\mathrm{SO} 2$ & -294.3 & $\mathrm{CH} 3$ & 149.0 & NH3 & -38.9 & $\mathrm{SiH} 3$ & 202.9 & $\mathrm{C} 2 \mathrm{H} 2$ & 228.6 \\
\hline $\mathrm{H} 2 \mathrm{O} 2$ & -130.0 & $\mathrm{H} 2 \mathrm{~S} 2$ & 16.0 & $\mathrm{H} 2 \mathrm{CN}$ & 189.0 & $\mathrm{H} 2 \mathrm{CO}$ & -104.7 & $\mathrm{SiCH} 2$ & ...... \\
\hline $\mathrm{H} 2 \mathrm{CS}$ & 105.0 & $\mathrm{H} 2 \mathrm{SiO}$ & $\ldots \ldots$ & $\mathrm{C} 3 \mathrm{H}$ & 602.5 & $\mathrm{SiC} 2 \mathrm{H}$ & $\ldots \ldots$ & $\mathrm{HCCP}$ & ....... \\
\hline $\mathrm{C} 4$ & 1052.0 & $\mathrm{C} 3 \mathrm{~N}$ & $\ldots \ldots$ & $\mathrm{C} 3 \mathrm{O}$ & $\ldots \ldots$ & $\mathrm{SiC} 3$ & $\ldots \ldots$ & $\mathrm{C} 3 \mathrm{P}$ & $\ldots \ldots$ \\
\hline C3S & 567.0 & $\mathrm{CH} 4$ & -66.8 & $\mathrm{SiH} 4$ & 46.0 & $\mathrm{C} 2 \mathrm{H} 3$ & 262.2 & $\mathrm{CH} 2 \mathrm{NH}$ & 110.0 \\
\hline SiCH3 & $\ldots \ldots$ & $\mathrm{CH} 2 \mathrm{PH}$ & $\ldots \ldots$ & $\mathrm{H} 2 \mathrm{CCC}$ & 512.0 & $\mathrm{C} 3 \mathrm{H} 2$ & $\ldots \ldots$ & $\mathrm{CH} 2 \mathrm{CN}$ & 245.0 \\
\hline $\mathrm{CH} 2 \mathrm{CO}$ & -44.6 & $\mathrm{SiC} 2 \mathrm{H} 2$ & $\ldots \ldots$ & $\mathrm{CHOOH}$ & -378.8 & $\mathrm{C} 4 \mathrm{H}$ & $\ldots \ldots$ & HC3N & 351.0 \\
\hline $\mathrm{SiC} 3 \mathrm{H}$ & $\ldots \ldots$ & C5 & 1081.0 & $\mathrm{SiC} 4$ & ....... & $\mathrm{C} 4 \mathrm{P}$ & ....... & $\mathrm{C} 4 \mathrm{~S}$ & \\
\hline $\mathrm{C} 2 \mathrm{H} 4$ & 60.7 & СH3OH & -190.7 & $\mathrm{C} 3 \mathrm{H} 3$ & 343.0 & CH3CN & 81.0 & $\mathrm{C} 4 \mathrm{H} 2$ & 440.0 \\
\hline $\mathrm{C} 5 \mathrm{H}$ & $\ldots \ldots$ & $\mathrm{C} 6$ & 1312.0 & $\mathrm{C} 5 \mathrm{~N}$ & $\ldots \ldots$ & $\mathrm{C} 2 \mathrm{H} 5$ & 130.0 & $\mathrm{C} 3 \mathrm{H} 4$ & 195.1 \\
\hline CH3CHO & -155.0 & H3C3N & 184.0 & $\mathrm{C} 5 \mathrm{H} 2$ & 723.0 & $\mathrm{C} 6 \mathrm{H}$ & $\ldots \ldots$ & $\mathrm{HC} 5 \mathrm{~N}$ & \\
\hline $\mathrm{C} 7$ & 1325.0 & $\mathrm{C} 2 \mathrm{H} 6$ & -69.1 & HCOOCH3 & -355.5 & $\mathrm{H} 3 \mathrm{C} 4 \mathrm{~N}$ & 338.0 & $\mathrm{C} 6 \mathrm{H} 2$ & 652.0 \\
\hline $\mathrm{C} 7 \mathrm{H}$ & $\ldots \ldots$ & $\mathrm{C} 8$ & 1487.0 & $\mathrm{C} 7 \mathrm{~N}$ & $\ldots \ldots$ & CH3OCH3 & -166.3 & $\mathrm{C} 2 \mathrm{H} 5 \mathrm{OH}$ & -217.1 \\
\hline $\mathrm{C} 5 \mathrm{H} 4$ & 425.0 & $\mathrm{C} 7 \mathrm{H} 2$ & 933.0 & $\mathrm{C} 8 \mathrm{H}$ & $\ldots \ldots$ & $\mathrm{HC} 7 \mathrm{~N}$ & $\ldots \ldots$ & $\mathrm{C} 9$ & 1554.0 \\
\hline $\mathrm{C} 2 \mathrm{H} 6 \mathrm{CO}$ & -217.2 & $\mathrm{H} 3 \mathrm{C} 6 \mathrm{~N}$ & $\ldots \ldots$ & $\mathrm{C} 8 \mathrm{H} 2$ & 864.0 & $\mathrm{C} 9 \mathrm{H}$ & ....... & $\mathrm{C} 9 \mathrm{~N}$ & $\ldots \ldots$ \\
\hline $\mathrm{C} 7 \mathrm{H} 4$ & $\ldots \ldots$ & $\mathrm{C} 9 \mathrm{H} 2$ & 1142.0 & HC9N & $\ldots \ldots$ & $\mathrm{H} 3 \mathrm{C} 8 \mathrm{~N}$ & ...... & $\mathrm{H}-$ & 143.2 \\
\hline $\mathrm{C}-$ & 589.3 & $\mathrm{O}-$ & 105.4 & $\mathrm{~S}-$ & 75.0 & $\mathrm{OH}-$ & -137.7 & $\mathrm{CN}-$ & 63.6 \\
\hline $\mathrm{H}+$ & 1528.0 & $\mathrm{He}+$ & 2372.0 & $\mathrm{C}+$ & 1797.6 & $\mathrm{~N}+$ & 1873.1 & $\mathrm{O}+$ & 1560.7 \\
\hline $\mathrm{Na}+$ & 603.4 & $\mathrm{Mg}+$ & 884.2 & $\mathrm{Si}+$ & 1233.0 & $\mathrm{P}+$ & 1328.0 & $\mathrm{~S}+$ & 1272.0 \\
\hline $\mathrm{Cl}+$ & 1371.0 & $\mathrm{Fe}+$ & 1173.0 & $\mathrm{H} 2+$ & 1488.3 & $\mathrm{HeH}+$ & 1352.0 & $\mathrm{CH}+$ & 1619.1 \\
\hline $\mathrm{NH}+$ & 1678.1 & $\mathrm{OH}+$ & 1292.7 & $\mathrm{SiH}+$ & 1136.2 & $\mathrm{PH}+$ & 1219.0 & $\mathrm{HS}+$ & 1137.0 \\
\hline $\mathrm{HCl}+$ & 1137.7 & $\mathrm{C} 2+$ & 1992.0 & $\mathrm{CN}+$ & 1796.3 & $\mathrm{CO}+$ & 1238.3 & $\mathrm{SiC}+$ & \\
\hline $\mathrm{CP}+$ & 1529.0 & $\mathrm{CS}+$ & 1356.0 & $\mathrm{CCl}+$ & 1243.0 & $\mathrm{~N} 2+$ & 1503.3 & $\mathrm{NO}+$ & 984.0 \\
\hline $\mathrm{SiN}+$ & ...... & $\mathrm{PN}+$ & 1249.0 & $\mathrm{NS}+$ & 1119.0 & $\mathrm{O} 2+$ & 1164.7 & $\mathrm{SiO}+$ & 1001.2 \\
\hline $\mathrm{PO}+$ & 778.0 & $\mathrm{SO}+$ & 1000.7 & $\mathrm{ClO}+$ & 1158.0 & $\mathrm{SiS}+$ & ...... & $\mathrm{S} 2+$ & 1031.0 \\
\hline $\mathrm{H} 3+$ & 1107.0 & $\mathrm{CH} 2+$ & 1386.0 & $\mathrm{NH} 2+$ & 1266.4 & $\mathrm{H} 2 \mathrm{O}+$ & 977.9 & $\mathrm{SiH} 2+$ & 1155.2 \\
\hline PH2+ & 1090.0 & $\mathrm{H} 2 \mathrm{~S}+$ & 991.0 & $\mathrm{H} 2 \mathrm{Cl}+$ & 867.0 & $\mathrm{C} 2 \mathrm{H}+$ & 1689.0 & $\mathrm{HCN}+$ & 1448.0 \\
\hline $\mathrm{HCO}+$ & 825.6 & $\mathrm{HOC}+$ & 963.0 & $\mathrm{HCSi}+$ & ....... & $\mathrm{HCP}+$ & 1208.0 & $\mathrm{HCS}+$ & 1018.0 \\
\hline $\mathrm{N} 2 \mathrm{H}+$ & 1035.5 & $\mathrm{HNO}+$ & 1074.4 & $\mathrm{HNSi}+$ & $\ldots \ldots$ & $\mathrm{HPN}+$ & $\ldots \ldots$ & $\mathrm{HNS}+$ & $\ldots \ldots$ \\
\hline $\mathrm{O} 2 \mathrm{H}+$ & 1108.5 & $\mathrm{SiOH}+$ & $\ldots \ldots$ & $\mathrm{HPO}+$ & $\ldots \ldots$ & $\mathrm{HSO}+$ & ....... & HSiS+ & ...... \\
\hline $\mathrm{S} 2 \mathrm{H}+$ & $\ldots \ldots$ & $\mathrm{C} 3+$ & 2004.0 & $\mathrm{CCN}+$ & 1715.0 & $\mathrm{CNC}+$ & 1620.0 & $\mathrm{C} 2 \mathrm{O}+$ & ...... \\
\hline $\mathrm{SiC} 2+$ & 1594.0 & $\mathrm{CCP}+$ & & $\mathrm{C} 2 \mathrm{~S}+$ & ...... & $\mathrm{NCO}+$ & 1289.0 & SiNC+ & \\
\hline $\mathrm{CO} 2+$ & 935.7 & $\mathrm{OCS}+$ & 936.0 & $\mathrm{NO} 2+$ & 977.0 & $\mathrm{SO} 2+$ & 894.0 & CH3+ & 1098.0 \\
\hline NH3+ & 941.0 & $\mathrm{H} 3 \mathrm{O}+$ & 597.0 & SiH3+ & 992.0 & PH3+ & 966.0 & H3S+ & 797.0 \\
\hline $\mathrm{C} 2 \mathrm{H} 2+$ & 1328.5 & $\mathrm{HCNH}+$ & 947.0 & $\mathrm{H} 2 \mathrm{NC}+$ & 1109.0 & $\mathrm{H} 2 \mathrm{CO}+$ & 944.5 & $\mathrm{SiCH} 2+$ & $\ldots \ldots$ \\
\hline PCH2+ & $\ldots \ldots$ & $\mathrm{H} 2 \mathrm{CS}+$ & 1006.0 & $\mathrm{H} 2 \mathrm{CCl}+$ & 962.1 & $\mathrm{H} 2 \mathrm{NO}+$ & 939.7 & SiNH2+ & 889.9 \\
\hline $\mathrm{PNH} 2+$ & $\ldots \ldots$ & $\mathrm{H} 2 \mathrm{SiO}+$ & $\ldots \ldots$ & $\mathrm{H} 2 \mathrm{PO}+$ & ....... & $\mathrm{H} 2 \mathrm{~S} 2+$ & 913.0 & $\mathrm{C} 3 \mathrm{H}+$ & 1593.0 \\
\hline $\mathrm{CCNH}+$ & 1531.0 & $\mathrm{HC} 2 \mathrm{O}+$ & 1096.0 & $\mathrm{C} 2 \mathrm{HO}+$ & $\ldots \ldots$ & $\mathrm{SiC} 2 \mathrm{H}+$ & $\ldots \ldots$ & $\mathrm{PC} 2 \mathrm{H}+$ & $\ldots \ldots$ \\
\hline $\mathrm{HC} 2 \mathrm{~S}+$ & $\ldots \ldots$ & $\mathrm{HNCO}+$ & 1015.0 & $\mathrm{SiNCH}+$ & $\ldots \ldots$ & $\mathrm{HCO} 2+$ & 589.0 & HOCS+ & 757.0 \\
\hline $\mathrm{HSiO} 2+$ & $\ldots \ldots$ & HSO2+ & 597.0 & $\mathrm{C} 4+$ & 2187.0 & $\mathrm{C} 3 \mathrm{~N}+$ & $\ldots \ldots$ & $\mathrm{C} 3 \mathrm{O}+$ & $\ldots \ldots$ \\
\hline SiC3+ & $\ldots \ldots$ & $\mathrm{C} 3 \mathrm{~S}+$ & $\ldots \ldots$ & $\mathrm{C} 2 \mathrm{~N} 2+$ & 1594.8 & $\mathrm{CH} 4+$ & 1140.0 & NH4+ & 630.0 \\
\hline $\mathrm{SiH} 4+$ & 1170.0 & C2H3+ & 1120.9 & $\mathrm{H} 3 \mathrm{CO}+$ & 703.0 & SiCH3+ & 977.0 & PCH3+ & ...... \\
\hline $\mathrm{H} 3 \mathrm{CS}+$ & 901.0 & PNH3+ & & $\mathrm{H} 3 \mathrm{SiO}+$ & $\ldots \ldots$ & $\mathrm{H} 3 \mathrm{~S} 2+$ & $\ldots \ldots$ & $\mathrm{C} 3 \mathrm{H} 2+$ & 1381.0 \\
\hline $\mathrm{CH} 2 \mathrm{CN}+$ & 1214.0 & $\mathrm{CH} 2 \mathrm{CO}+$ & 882.7 & $\mathrm{SiC} 2 \mathrm{H} 2+$ & $\ldots \ldots$ & $\mathrm{PC} 2 \mathrm{H} 2+$ & $\ldots \ldots$ & $\mathrm{C} 4 \mathrm{H}+$ & 1805.0 \\
\hline
\end{tabular}


Table 2. continued

\begin{tabular}{|c|c|c|c|c|c|c|c|c|c|}
\hline $\mathrm{HC}$ & 174.0 & $\mathrm{~b}+$ & 971.0 & $\mathrm{H}+$ & & $\mathrm{H}+$ & & + & $\cdots \cdots$ \\
\hline $\mathrm{Cs}$ & 0 & & & & & & & & \\
\hline $5+$ & 5.0 & + & 917.0 & $4+$ & 74.0 & $\mathrm{~N}+$ & 745.0 & $\mathrm{NH} 2+$ & $\ldots$ \\
\hline $\mathrm{CH} 3 \mathrm{OH}+$ & 856.2 & $\mathrm{CH} 4+$ & 1015.0 & PCH4+ & 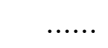 & $\mathrm{H} 3+$ & 1075.0 & $\mathrm{H} 2 \mathrm{C} 3 \mathrm{H}+$ & $\ldots \ldots$ \\
\hline $\mathrm{CH} 3$ & 258.0 & $\mathrm{H} 3 \mathrm{CO}+$ & 653.0 & $\mathrm{C} 2 \mathrm{H} 3+$ & & $2 \mathrm{H} 3+$ & $\ldots \ldots$ & $\mathrm{CHOOH} 2+$ & 403.0 \\
\hline $\mathrm{C} 4 \mathrm{H}$ & 422.0 & $\mathrm{H} 2 \mathrm{C} 3 \mathrm{~N}+$ & 1127.5 & $\mathrm{C} 3 \mathrm{H} 2 \mathrm{O}+$ & 157.0 & $\mathrm{SiC} 3 \mathrm{H} 2+$ & ...... & $\mathrm{C} 5 \mathrm{H}+$ & $\ldots$ \\
\hline $\mathrm{HC}$ & $\ldots$ & $\mathrm{SiC} 4 \mathrm{H}+$ & $\ldots$. & $\mathrm{PC} 4 \mathrm{H}+$ & & S+ & $\ldots \ldots$ & $\mathrm{Ct}$ & $\ldots$ \\
\hline & ....... & $\mathrm{C} 2 \mathrm{H} 5+$ & 914.0 & $\mathrm{OH} 2+$ & 567.0 & $4+$ & 1194.5 & $\mathrm{C} 2 \mathrm{~N}+$ & 817.0 \\
\hline CH3CHO+ & 831.9 & PC2H4+ & $\ldots$ & C4H3+ & 1217.0 & $\mathrm{H} 3 \mathrm{C} 3 \mathrm{O}+$ & 751.0 & $\mathrm{C} 5 \mathrm{H} 2+$ & \\
\hline $\mathrm{H} 2 \mathrm{C}$ & & $\mathrm{PC} 4 \mathrm{H} 2+$ & $\ldots$. & $\mathrm{C} 6 \mathrm{H}+$ & & $\mathrm{HC} 5 \mathrm{~N}+$ & .... & & 2299.0 \\
\hline $\mathrm{C} 2 \mathrm{H} 6+$ & 043.0 & $\mathrm{C} 3 \mathrm{H} 5+$ & 969.0 & $\mathrm{C} 2 \mathrm{H} 5 \mathrm{O}+$ & 583.0 & $\mathrm{H} 5 \mathrm{C} 2 \mathrm{O}+$ & $\ldots$ & $\mathrm{H} 4 \mathrm{C} 3 \mathrm{~N}+$ & 817.0 \\
\hline COOCH4+ & 688.0 & C5H3+ & $\ldots \ldots$ & $\mathrm{H} 3 \mathrm{C} 4 \mathrm{~N}+$ & $\cdot \cdot$ & $\mathrm{C} 6 \mathrm{H} 2+$ & 1569.0 & & $\ldots \ldots$ \\
\hline $\mathrm{C} 7 \mathrm{H}+$ & & $\mathrm{C} 8+$ & & $\mathrm{C} 7 \mathrm{~N}+$ & & $\mathrm{C} 2 \mathrm{H} 7+$ & 856.4 & $\mathrm{C} 2 \mathrm{H} 5 \mathrm{OH}+$ & 793.1 \\
\hline $\mathrm{C} 2 \mathrm{H} 6 \mathrm{O}+$ & 801.0 & $\mathrm{C} 4 \mathrm{H} 5+$ & 076.0 & $\mathrm{H} 5 \mathrm{C} 2 \mathrm{O} 2+$ & 386.0 & C5H4+ & 1332.0 & $\mathrm{H} 4 \mathrm{C} 4 \mathrm{~N}+$ & $\ldots$. \\
\hline $\mathrm{C} 6 \mathrm{H}$ & & $\mathrm{H} 3 \mathrm{C} 5 \mathrm{~N}+$ & & & & & $\cdots$ & $\mathrm{HC}$ & $\cdots$ \\
\hline $\mathrm{C} 9+$ & 2451.0 & $\mathrm{C} 2 \mathrm{H} 6 \mathrm{OH}+$ & 542.0 & $\mathrm{C} 2 \mathrm{H} 7 \mathrm{O}+$ & 507.0 & $\mathrm{C} 2 \mathrm{H} 6 \mathrm{CO}+$ & 719.7 & $\mathrm{C} 5 \mathrm{H} 5+$ & 1132.0 \\
\hline $\mathrm{C} 6 \mathrm{H} 4+$ & 1400.0 & C7H3+ & ...... & $\mathrm{C} 8 \mathrm{H} 2+$ & 1741.0 & $\mathrm{H} 2 \mathrm{C} 7 \mathrm{~N}+$ & .. & $\mathrm{C} 9 \mathrm{H}+$ & $\ldots \ldots$ \\
\hline & & $\mathrm{C} 9 \mathrm{~N}+$ & $\ldots \ldots$ & $\mathrm{C} 3 \mathrm{H} 6 \mathrm{OH}+$ & 490.0 & $\mathrm{C} 6 \mathrm{H} 5+$ & 1141.3 & $\mathrm{C} 7 \mathrm{H} 4$ & $\cdots$ \\
\hline $\mathrm{H} 4 \mathrm{C}$ & $\cdots \cdots$ & C8H3+ & $\ldots \ldots$ & $\mathrm{H} 3 \mathrm{C} 7 \mathrm{~N}+$ & $\cdots$ & $\mathrm{C} 9 \mathrm{H} 2+$ & $\cdots$ & $\mathrm{HC} 9$ & ..... \\
\hline & $\ldots \ldots$ & $\mathrm{C} 8 \mathrm{H} 4+$ & $\ldots$ & & & $\mathrm{H} 2 \mathrm{C} 9 \mathrm{~N}+$ & $\ldots \ldots$ & & $\cdots$ \\
\hline & 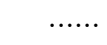 & QNT & 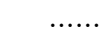 & $\mathrm{H}^{3}$ & & & ...... & & \\
\hline
\end{tabular}

Notes: These data were compiled from the NIST-JANAF tables, mostly by H.-H. Lee (Ohio State University), with the exception of the heats of formation for the carbon chains $\mathrm{C}_{n}, n=2-7$, which come from Gingerich et al. (1994), and for the carbenes, $\mathrm{H}_{2} \mathrm{C}_{n}$, which come from Bettens et al. (1995). The heat of formation for CH2NH is taken from the NIST Webbook (Afeefy et al. 2000), and for C2S and C3S from J.R. Flores (private communication). These three entries are values at $298 \mathrm{~K}$ not $0 \mathrm{~K}$.

where $\alpha$ is the cosmic-ray ionisation rate, $\gamma$ is the probability per cosmic-ray ionisation that the appropriate photoreaction takes place, and $\omega$ is the dust grain albedo in the far ultraviolet (typically 0.6 at $150 \mathrm{~nm}$ ). We note that because $\mathrm{CO}$ is destroyed by the line absorption, its rate of destruction is sensitive to its rotational level populations. To account for this we have included a temperaturedependence in the calculation of the rate coefficient.

For interstellar photoreactions $(\mathrm{R} 2=\mathrm{PHOTON})$, the rate is derived as:

$k=\alpha \exp \left(-\gamma A_{\mathrm{V}}\right) \quad \mathrm{s}^{-1}$

where $\alpha$ represents the rate in the unshielded interstellar ultraviolet radiation field, $A_{\mathrm{V}}$ is the extinction at visible wavelengths caused by interstellar dust, and $\gamma$ is the parameter used to take into account the increased extinction of dust at ultraviolet wavelengths.

\subsection{General form of the reaction set}

We have re-organised the order of reactions in this release. The reactions are divided into 14 categories or types, which are grouped together in the database. Table 5 summarizes these categories, along with their position in the database. Within each category, the reactions are listed by increasing total molar mass (total mass of the reactants).

- Photoprocesses: the temperature flags of the photoprocesses, which are just an artifact from the data revision software, are, of course, irrelevant. Also, the unshielded rates shown are valid only for the ISM UV field (from Draine 1978). These photorates can be rescaled to a stellar radiation field or calculated from cross sections when available (see Table 7 and Eq. (5));

- Neutral-neutral reactions: the reactions that were already present in the former UMIST Database have had their temperature dependence reviewed and temperature range defined when known. When not known, an arbitrary range of 10 to $300 \mathrm{~K}$ has been attributed, since these reactions were originally defined for these low temperatures;

- Cosmic-Ray reactions: reactions with CRPHOT (cosmic ray photons) and CRP (cosmic ray protons) have been left unchanged;

- Sundries: this section gathers all the reactions that cannot be classified by any of the other types because they are a combination of at least two different types.

\subsection{Alterations present in this release}

- A major change brought to the database is the inclusion of the temperature dependence and temperature range for all the reactions. The maximum temperature range used in the database has been arbitrarily defined from 10 to $41000 \mathrm{~K}$. Where explicit information is not available, the rate coefficients have been attributed a 
Table 3. Permanent electric dipole moments in Debye of the neutral molecules

\begin{tabular}{|c|c|c|c|c|c|}
\hline Species & $\mu_{\mathrm{D}}$ & Species & $\mu_{\mathrm{D}}$ & Species & $\mu_{\mathrm{D}}$ \\
\hline $\mathrm{H} 2$ & 0 & $\mathrm{CH}$ & 1.46 & $\mathrm{NH}$ & 1.3 \\
\hline $\mathrm{OH}$ & 1.66 & $\mathrm{SiH}$ & 0.12 & $\mathrm{PH}$ & 0.64 \\
\hline HS & 0.76 & $\mathrm{HCl}$ & 1.08 & $\mathrm{C} 2$ & 0 \\
\hline $\mathrm{CN}$ & 1.45 & $\mathrm{CO}$ & 0.112 & $\mathrm{SiC}$ & 1.7 \\
\hline $\mathrm{CP}$ & 0.86 & CS & 1.96 & $\mathrm{CCl}$ & $<0.65$ \\
\hline $\mathrm{N} 2$ & 0 & $\mathrm{NO}$ & 0.153 & $\mathrm{SiN}$ & $\sim 2.3$ \\
\hline PN & 2.75 & NS & 1.81 & $\mathrm{O} 2$ & 0 \\
\hline $\mathrm{SiO}$ & 3.1 & $\mathrm{PO}$ & 1.88 & $\mathrm{SO}$ & 1.55 \\
\hline $\mathrm{ClO}$ & 1.24 & $\mathrm{SiS}$ & 1.73 & $\mathrm{~S} 2$ & 0 \\
\hline $\mathrm{CH} 2$ & 0.57 & NH2 & 1.83 & $\mathrm{H} 2 \mathrm{O}$ & 1.85 \\
\hline $\mathrm{SiH} 2$ & 0.18 & PH2 & $\ldots$ & $\mathrm{H} 2 \mathrm{~S}$ & 0.97 \\
\hline $\mathrm{C} 2 \mathrm{H}$ & 0.8 & $\mathrm{HCN}$ & 2.98 & $\mathrm{HNC}$ & 2.7 \\
\hline $\mathrm{HCO}$ & $\sim 1.0$ & $\mathrm{HCSi}$ & $\ldots$ & $\mathrm{HCP}$ & 0.3 \\
\hline HCS & $\ldots$ & HNO & 1.67 & HNSi & 0.16 \\
\hline $\mathrm{O} 2 \mathrm{H}$ & 2.09 & HPO & 2.33 & HS2 & $\ldots$ \\
\hline C3 & 0 & $\mathrm{CCN}$ & 0.6 & $\mathrm{CCO}$ & 1.3 \\
\hline $\mathrm{SiC} 2$ & 2.39 & $\mathrm{CCP}$ & $\ldots$ & $\mathrm{C} 2 \mathrm{~S}$ & 2.8 \\
\hline OCN & 0.64 & $\mathrm{SiNC}$ & 2.03 & $\mathrm{CO} 2$ & 0 \\
\hline OCS & 0.71 & $\mathrm{~N} 2 \mathrm{O}$ & 0.16 & NO2 & 0.32 \\
\hline $\mathrm{SiO} 2$ & $\sim 0.5$ & $\mathrm{SO} 2$ & 1.63 & CH3 & 0 \\
\hline NH3 & 1.47 & SiH3 & 0 & $\mathrm{C} 2 \mathrm{H} 2$ & 0 \\
\hline $\mathrm{H} 2 \mathrm{O} 2$ & 1.57 & $\mathrm{H} 2 \mathrm{~S} 2$ & 1.2 & $\mathrm{H} 2 \mathrm{CN}$ & 2.54 \\
\hline $\mathrm{H} 2 \mathrm{CO}$ & 2.33 & $\mathrm{SiCH} 2$ & $\ldots$ & $\mathrm{H} 2 \mathrm{CS}$ & 1.65 \\
\hline $\mathrm{H} 2 \mathrm{SiO}$ & $\ldots$ & 1-C3H & 3.1 & $\mathrm{SiC} 2 \mathrm{H}$ & 1.4 \\
\hline $\mathrm{HCCP}$ & 0 & $\mathrm{C} 4$ & 0 & $\mathrm{C} 3 \mathrm{~N}$ & 2.2 \\
\hline $\mathrm{C} 3 \mathrm{O}$ & 2.39 & $\mathrm{SiC} 3$ & $\ldots$ & C3P & $\ldots$ \\
\hline C3S & 3.7 & $\mathrm{CH} 4$ & 0 & $\mathrm{SiH} 4$ & 0 \\
\hline $\mathrm{C} 2 \mathrm{H} 3$ & $\sim 1.5$ & $\mathrm{CH} 2 \mathrm{NH}$ & 2.02 & $\mathrm{SiCH} 3$ & $\ldots$ \\
\hline $\mathrm{CH} 2 \mathrm{PH}$ & $\ldots$ & c-C3H2 & 3.4 & $\mathrm{CH} 2 \mathrm{CN}$ & 1.62 \\
\hline $\mathrm{CH} 2 \mathrm{CO}$ & 1.42 & $\mathrm{SiC} 2 \mathrm{H} 2$ & 2.5 & $\mathrm{CHOOH}$ & 1.41 \\
\hline $\mathrm{C} 4 \mathrm{H}$ & 0.9 & HC3N & 3.6 & $\mathrm{SiC} 3 \mathrm{H}$ & $\ldots$ \\
\hline C5 & 0 & $\mathrm{SiC} 4$ & 6.3 & $\mathrm{C} 4 \mathrm{P}$ & $\ldots$ \\
\hline $\mathrm{C} 4 \mathrm{~S}$ & $\sim 3.0$ & $\mathrm{C} 2 \mathrm{H} 4$ & 0 & $\mathrm{CH} 3 \mathrm{OH}$ & 1.7 \\
\hline $\mathrm{C} 3 \mathrm{H} 3$ & 4.0 & $\mathrm{CH} 3 \mathrm{CN}$ & 3.92 & $\mathrm{H} 2 \mathrm{CCCC}$ & 4.5 \\
\hline $\mathrm{C} 5 \mathrm{H}$ & 4.3 & C6 & 0 & $\mathrm{C} 5 \mathrm{~N}$ & $\sim 2.7$ \\
\hline $\mathrm{C} 2 \mathrm{H} 5$ & $\ldots$ & $\mathrm{C} 3 \mathrm{H} 4[\mathrm{CH} 3 \mathrm{CCH}]$ & 0.78 & $\mathrm{CH} 3 \mathrm{CHO}$ & 2.69 \\
\hline $\mathrm{H} 3 \mathrm{C} 3 \mathrm{~N}$ [CH2CHCN] & 3.89 & $\mathrm{C} 5 \mathrm{H} 2$ & 2.5 & $\mathrm{C} 6 \mathrm{H}$ & 5.0 \\
\hline $\mathrm{HC} 5 \mathrm{~N}$ & 4.33 & $\mathrm{C} 7$ & 0 & $\mathrm{C} 2 \mathrm{H} 6$ & 0 \\
\hline HCOOCH3 & 1.77 & $\mathrm{H} 3 \mathrm{C} 4 \mathrm{~N}$ [CH3C3N] & 4.91 & $\mathrm{C} 6 \mathrm{H} 2$ & 0 \\
\hline $\mathrm{C} 7 \mathrm{H}$ & 4.5 & $\mathrm{C} 8$ & 0 & $\mathrm{C} 7 \mathrm{~N}$ & 3.0 \\
\hline CH3OCH3 & 1.3 & $\mathrm{C} 2 \mathrm{H} 5 \mathrm{OH}$ & 1.44 & $\mathrm{C} 5 \mathrm{H} 4[\mathrm{CH} 3 \mathrm{C} 4 \mathrm{H}]$ & 1.21 \\
\hline $\mathrm{C} 7 \mathrm{H} 2$ & 2.5 & $\mathrm{C} 8 \mathrm{H}$ & 5.0 & $\mathrm{HC} 7 \mathrm{~N}$ & 4.62 \\
\hline $\mathrm{C} 9$ & 0 & $\mathrm{C} 2 \mathrm{H} 6 \mathrm{CO}$ & 2.8 & $\mathrm{H} 3 \mathrm{C} 6 \mathrm{~N}[\mathrm{CH} 3 \mathrm{C} 5 \mathrm{~N}]$ & 5.75 \\
\hline $\mathrm{C} 8 \mathrm{H} 2$ & 0 & $\mathrm{C} 9 \mathrm{H}$ & 4.7 & $\mathrm{C} 9 \mathrm{~N}$ & 3.3 \\
\hline $\mathrm{C} 7 \mathrm{H} 4[\mathrm{CH} 3 \mathrm{C} 6 \mathrm{H}]$ & 1.5 & $\mathrm{C} 9 \mathrm{H} 2$ & 2.5 & $\mathrm{HC} 9 \mathrm{~N}$ & 4.84 \\
\hline $\mathrm{H} 3 \mathrm{C} 8 \mathrm{~N}$ [CH3C7N] & 5.47 & & & & \\
\hline
\end{tabular}

Notes: c-C3H = 2.4D; H2CCC = 4.1D; HCCCCH = 0D; H2C3H(propargyl) = 0.14D;

C3S from Suernam \& Lovas (1994); CH3C3N and CH3C5N from Botschwina et al. (1994);

H2CCCC from Oswald \& Botschwina (1995); C3N from Pauzat et al. (1991);

C5N from Botschwina (1996); HCN, HC3N, HC7N and HC9N from Botschwina \& Horn (1997). 
Table 4. List of the database reaction reference flags and their correspondance with the bibliographical references. Note: Any digit-only flag found in the database is the same flag as that used for referencing reactions in Anicich \& Huntress (1986) and Anicich (1993)

\begin{tabular}{|c|c|c|c|}
\hline Flags & References & Flags & References \\
\hline AA83 & Alge et al. 1983 & AM90 & Adams et al. 1990 \\
\hline AS84 & Adams et al. 1984 & AS95 & Andreazza et al. 1995 \\
\hline AS97 & Andreazza and Singh 1997 & BA84 & Barlow 1984 \\
\hline BC92 & Baulch et al. 1992 & BH83 & Becker and Hong 1983 \\
\hline BO90 & Bohme 1990 & BS97 & Brownsword and Sims 1997 \\
\hline BW87 & Bohme et al. 1987 & CH82 & Copp et al. 1982 \\
\hline CH94 & Clary et al. 1994 & DA97 & Decker and Adams 1997 \\
\hline DD90 & Dalgarno et al. 1990 & DF86 & Dheandanoo et al. 1986 \\
\hline DM77 & Mann 1977 & FA80 & Field et al. 1980 \\
\hline DPFR & \multicolumn{3}{|c|}{ Donnelly and Pasternack 1979, Filseth et al. 1979, Reisler et al. 1980} \\
\hline FO91 & Forst 1991 & FS91 & Frost et al. 1991 \\
\hline FV86 & Federer et al. 1986 & GA89 & Giles et al. 1989 \\
\hline GH92 & Gerlich and Horning 1992 & GI90 & Giles 1990 \\
\hline GL87 & Gredel et al. 1987 & GL89 & Gredel et al. 1989 \\
\hline HA 89 & Herbst, Adams et al. 1989 & HA90 & Herd et al. 1990 \\
\hline HA93 & Haider and Husain 1993a & HD89 & Herbst, DeFrees and Koch 1989 \\
\hline HE85 & Herbst 1985 & HE87 & Herbst 1987 \\
\hline HG90 & Herbst, Giles and Smith 1990 & HG93 & Harding et al. 1993 \\
\hline HН93 & Haider and Husain 1993b & HL86 & Herbst and Leung 1986 \\
\hline HL89 & Herbst and Leung 1989 & HL90 & Herbst and Leung 1990 \\
\hline HL97 & Herbst and Lee 1997 & HM89 & Herbst, Millar et al. 1989 \\
\hline HN79 & Husain and Norris 1979 & HT00 & Herbst et al. 2000 \\
\hline IS89 & Smith I.W.M. 1989 & KD93 & Kimura and Dalgarno 1993 \\
\hline KM89 & Karpas and Meot-ner 1989 & LG88 & Leen and Graff 1988 \\
\hline LH84 & Leung et al. 1984 & LL98 & Larson et al. 1998 \\
\hline MA85 & Millar et al. 1985 & MA86 & Millar, Adams et al. 1986 \\
\hline MB73 & MacGregor \& Berry 1973 & MB87 & Millar et al. 1987 \\
\hline MD83 & Mitchell and Deveau 1983 & MH90 & Millar and Herbst 1990 \\
\hline MH91 & Millar, Herbst and Charnley 1991 & MI84 & Mitchell 1984 \\
\hline MI90 & Mitchell 1990 & MI91 & Millar 1991 \\
\hline MM93 & Maluendes et al. 1993 & MN85 & Millar and Nejad 1985 \\
\hline MN89 & Marston et al. 1989 & MS99 & McEwan et al. 1999 \\
\hline NA99 & Nahar 1999 & NIST & Mallard et al. 1998 \\
\hline NM90 & Nesbitt et al. 1990 & NP97 & Nahar and Pradhan 1997 \\
\hline PD89 & Petuchowski et al. 1989 & PF92 & Petrie et al. 1992 \\
\hline PH80 & Prasad and Huntress 1980 & PH82 & Prasad and Huntress 1982 \\
\hline PR90 & Pineau des Forêts et al. 1990 & RA92 & Rawlings 1992 \\
\hline RJ91 & Roberge et al. 1991 & RW80 & Raksit and Warneck 1980 \\
\hline RW88 & Rawlings et al. 1988 & SA84 & Smith and Adams 1984 \\
\hline SA 88 & Smith, Adams et al. 1988 & SA92 & Sen et al. 1992 \\
\hline SM88 & Stief et al. 1988 & SM89 & Smith et al. 1989 \\
\hline SM93 & Smith M.A. 1993 & SQ93 & Sims et al. 1993 \\
\hline SQ94 & Sims et al. 1994 & SS92 & Smith et al. 1992 \\
\hline SS93 & Smith and Spanel 1993 & SS94 & Smith et al. 1994 \\
\hline SS99 & Stancil et al. 1999 & SY92 & Suzuki et al. 1992 \\
\hline TA84 & Thorne et al. 1984 & TE96 & Talbi et al. 1996 \\
\hline TH86 & Tsang and Hampson 1986 & VD87 & van Dishoeck 1987 \\
\hline VA99 & \multicolumn{3}{|c|}{ Vikor et al. 1999. The values of the total rates are from MI90 and AA83 (at $300 \mathrm{~K}$ ) } \\
\hline VD88 & van Dishoeck 1988 & VP83 & Viggiano and Paulson 1983 \\
\hline WB88 & Wlodek et al. 1988 & WM94 & Wilson et al. 1994 \\
\hline ZD89 & Zygelman et al. 1989 & & \\
\hline
\end{tabular}


range of $10-300 \mathrm{~K}$. However, if their " $\gamma$ " Arrhenius coefficient is too large, the lowest temperature has been defined as $T_{1}=\gamma / 30$ (in $\mathrm{K}$ ), and the largest temperature $T_{\mathrm{u}}$ has been arbitrarily taken as $41000 \mathrm{~K}$ (the value of such rates does not change significantly at higher temperatures), unless the rate's value becomes unphysical, in which case an appropriate upper temperature has been determined. Many Ion-Neutral and Ion-Ion reactions remain constant whatever the temperature, and where this is the case they have been arbitrarily defined from 10 to $41000 \mathrm{~K}$;

- Two new reaction types have been added, namely Termolecular reactions and Collider reactions, both of which become important at high density, typically above $10^{10} \mathrm{~cm}^{-3}$. Termolecular reactions are catalysed bimolecular reactions and the catalyst is named "M". The nature of the third body is not important in general because it is only used as a de-excitation energy carrier:

$$
\mathrm{X}+\mathrm{Y}+\mathrm{M} \rightarrow \mathrm{XY}+\mathrm{M}
$$

Collider reactions are collision-induced dissociations and the collider is also named "M" as its nature does not significantly alter the rate of the process:

$\mathrm{XY}+\mathrm{M} \rightarrow \mathrm{X}+\mathrm{Y}+\mathrm{M}$

- Because the reactions were chosen to be appropriate not only for the cold ISM, less discrimination has been operated so novel reactions have been added to all the types. The net result is a richer chemistry with multiproduct reactions;

- Tables of cross section data appropriate for photo processes have been gathered and are included in the electronic tables to allow study of chemistry in a variety of radiation fields. Table 7 gives further information on these cross sections.

\section{Other data}

\subsection{Deuterium chemistry}

A comprehensive description of deuterium chemistry requires that all D-bearing analogues of H-bearing species are included in a chemical model. This has the effect of approximately tripling the number of reactions in a model if it is to include deuterium. For our purposes, we have decided not to include a full deuterium chemistry in this release. Such a chemistry can be generated most efficiently using software but the detailed branching ratios adopted are a matter of individual choice at this time and we prefer to list only the most important reactions in Table 6 . Rodgers \& Millar (1996) have discussed many of the issues involved in generating a deuterium chemistry.
Table 5. List of the types of chemical reactions and their position in the database. The unclassifiable reactions are put in the category "Sundries". The first column XX is the abbreviation used for the types

\begin{tabular}{clr}
\hline XX & $\begin{array}{l}\text { Type of } \\
\text { reaction }\end{array}$ & $\begin{array}{c}\text { Position } \\
\text { (index "I") }\end{array}$ \\
\hline NN & Neutral - Neutral Reactions & $1-433$ \\
IN & Ion - Neutral Reactions & $434-2606$ \\
CE & Charge Exchange Reactions & $2607-3144$ \\
II & Ion - Ion Neutralisations & $3145-3175$ \\
DR & Dissociative Recombinations & $3176-3606$ \\
RR & Radiative Recombinations & $3607-3631$ \\
AD & Associative Detachments & $3632-3678$ \\
RA & Radiative Associations & $3679-3760$ \\
PH & Photoprocesses & $3761-3916$ \\
CP & Cosmic-Ray Proton Reacs (CRP) & $3917-3927$ \\
CR & Cosmic-Ray Photon Reacs (CRPHOT) & $3928-4059$ \\
CL & Collider Reactions & $4060-4077$ \\
TR & Termolecular Reactions & $4078-4107$ \\
- & Sundries & $4108-4113$ \\
\hline
\end{tabular}

\subsection{Cross sections for photoprocesses}

The necessity for having data on photo process cross sections arises from the fact that astrochemistry is not exclusive to the ISM but is now applied to circumstellar regions and comets as well. As a result, the rates of destruction of the species through exposure to the stellar radiation field can change by several orders of magnitude. For example, the photoionization rate of $\mathrm{He}^{0}$ in interstellar clouds is negligible, whereas in the radiation field of a nearby Wolf-Rayet star it can be as large as $10^{-5} \mathrm{~s}^{-1}$. Table 7 gives the list of the species and the data references compiled to date by us. The detailed cross-sections, which are available electronically, are given in megabarns $(1 \mathrm{Mb}=$ $\left.10^{-18} \mathrm{~cm}^{2}\right)$. The photorate, $\beta(X, r)\left(\mathrm{s}^{-1}\right)$, for species $X$ at a distance $r$ from a localised radiation source can be derived using Eq. (5):

$\beta(X, r)=\frac{10^{-26} w(r)}{h c} \int_{\lambda<\lambda_{0}}^{\lambda_{0}} \lambda \mathcal{F}_{\lambda} \sigma_{\lambda}(X) \mathrm{d} \lambda$

where $w(r)$ is the dilution factor at the distance $r$ from the source, $\lambda_{0}(\AA)$ is the photoprocess threshold wavelength, such that any photon with $\lambda<\lambda_{0}$ will take part to the process, $\mathcal{F}_{\lambda}$ is the source Spectral Energy Distribution in erg $\mathrm{cm}^{-2} \mathrm{~s}^{-1} \AA^{-1}, \sigma_{\lambda}(X)$ is the cross section (Mb) for the species $X$ at the wavelength $\lambda(\AA)$.

These data are not complete. Future work is aimed at updating these via the Opacity Project and other sources.

\subsection{Dipole moments}

At low temperatures, the rates of ion-molecule reaction rate coefficients can increase dramatically through iondipole interactions, which are particularly important for 
Table 6. Deuterium Reactions. NOTE: $a(b)$ stands for $a \times 10^{b}$. (D-resc) means that the equivalent H-only reaction rate has been rescaled by D reduced mass. ${ }^{*}$ implies that the exponential term in the rate expression has the form $\exp (-T / \gamma)$. References: as: Adams \& Smith 1985; cd: Crosswell \& Dalgarno 1985; dl: Dalgarno \& Lepp 1987; dmd: Dalgarno \& McDowell 1956; fp: Frommhold \& Pickett 1978; hasd: Herbst et al. 1987; kah: Karpas et al. 1979; lbh: Lee et al. 1996; ljb: Linder et al. 1995; lm: Larsson et al. 1996; pdf: Pineau des Forêts et al. 1986; rp: Ramaker \& Peek 1976; s: Schilke et al. 1992; saa: Smith et al. 1982a,b; smt: Sidhu et al. 1992; str: Strömholm et al. 1995; w: Watson 1976; zm: Zhang \& Miller 1989

\begin{tabular}{|c|c|c|c|c|c|c|c|c|c|c|}
\hline Reaction & & & & & & & $\alpha\left(\mathrm{cm}^{3} \mathrm{~s}^{-1}\right)$ & $\beta$ & $\gamma(\mathrm{K})$ & References \\
\hline $\mathrm{H}_{3}^{+}$ & + & HD & $\rightarrow$ & $\mathrm{H}_{2} \mathrm{D}^{+}$ & + & $\mathrm{H}_{2}$ & $1.5(-09)$ & & & lbh \\
\hline $\mathrm{H}_{2} \mathrm{D}^{+}$ & + & $\mathrm{H}_{2}$ & $\rightarrow$ & $\mathrm{H}_{3}^{+}$ & + & HD & $2.0(-09)$ & -0.8 & 230 & smt \\
\hline $\mathrm{CH}_{2}^{+}$ & + & $\mathrm{HD}$ & $\rightarrow$ & $\mathrm{CH}_{2} \mathrm{D}^{+}$ & + & $\mathrm{H}_{2}$ & $1.3(-09)$ & - & - & saa \\
\hline $\mathrm{CH}_{2} \mathrm{D}^{+}$ & + & $\mathrm{H}_{2}$ & $\rightarrow$ & $\mathrm{CH}_{2}^{+}$ & + & $\mathrm{HD}$ & $8.7(-10)$ & - & 370 & saa \\
\hline $\mathrm{C}_{2} \mathrm{H}_{2}^{+}$ & + & HD & $\rightarrow$ & $\mathrm{C}_{2} \mathrm{HD}^{+}$ & + & $\mathrm{H}_{2}$ & $1.0(-09)$ & - & - & hasd \\
\hline $\mathrm{C}_{2} \mathrm{HD}^{+}$ & + & $\mathrm{H}_{2}$ & $\rightarrow$ & $\mathrm{C}_{2} \mathrm{H}_{2}^{+}$ & + & HD & $2.5(-09)$ & - & 550 & hasd \\
\hline $\mathrm{D}^{+}$ & + & $\mathrm{H}_{2}$ & $\rightarrow$ & $\mathrm{H}^{+}$ & + & HD & $2.1(-09)$ & - & - & saa \\
\hline $\mathrm{H}^{+}$ & + & $\mathrm{HD}$ & $\rightarrow$ & $\mathrm{D}^{+}$ & + & $\mathrm{H}_{2}$ & $1.0(-09)$ & - & 464 & saa \\
\hline $\mathrm{D}^{+}$ & + & $\mathrm{H}$ & $\rightarrow$ & $\mathrm{H}^{+}$ & + & $\mathrm{D}$ & $1.0(-09)$ & - & - & $\mathrm{w}$ \\
\hline $\mathrm{H}^{+}$ & + & $\mathrm{D}$ & $\rightarrow$ & $\mathrm{D}^{+}$ & + & $\mathrm{H}$ & $1.0(-09)$ & - & 41 & $\mathrm{w}$ \\
\hline $\mathrm{H}_{3}^{+}$ & + & $\mathrm{D}$ & $\rightarrow$ & $\mathrm{H}_{2} \mathrm{D}^{+}$ & + & $\mathrm{H}$ & $1.0(-09)$ & - & - & as (estimate) \\
\hline $\mathrm{H}_{2} \mathrm{D}^{+}$ & + & $\mathrm{H}$ & $\rightarrow$ & $\mathrm{H}_{3}^{+}$ & + & $\mathrm{D}$ & $1.0(-09)$ & - & 632 & as (estimate) \\
\hline $\mathrm{HCO}^{+}$ & + & $\mathrm{D}$ & $\rightarrow$ & $\mathrm{DCO}^{+}$ & + & $\mathrm{H}$ & $1.0(-09)$ & - & - & as \\
\hline $\mathrm{DCO}^{+}$ & + & $\mathrm{H}$ & $\rightarrow$ & $\mathrm{HCO}^{+}$ & + & $\mathrm{D}$ & $2.2(-09)$ & - & 796 & as \\
\hline $\mathrm{N}_{2} \mathrm{H}^{+}$ & + & $\mathrm{D}$ & $\rightarrow$ & $\mathrm{N}_{2} \mathrm{D}^{+}$ & + & $\mathrm{H}$ & $1.0(-09)$ & - & - & as \\
\hline $\mathrm{N}_{2} \mathrm{D}^{+}$ & + & $\mathrm{H}$ & $\rightarrow$ & $\mathrm{N}_{2} \mathrm{H}^{+}$ & + & $\mathrm{D}$ & $2.2(-09)$ & - & 550 & as \\
\hline $\mathrm{OH}$ & + & $\mathrm{D}$ & $\rightarrow$ & $\mathrm{OD}$ & + & $\mathrm{H}$ & $1.3(-10)$ & - & - & $\mathrm{cd}$ \\
\hline OD & + & $\mathrm{H}$ & $\rightarrow$ & $\mathrm{OH}$ & + & $\mathrm{D}$ & $1.3(-10)$ & - & 810 & $\mathrm{~cd}$ \\
\hline $\mathrm{N}^{+}$ & + & $\mathrm{HD}$ & $\rightarrow$ & $\mathrm{ND}^{+}$ & + & $\mathrm{H}$ & $3.2(-10)$ & - & 16 & $\mathrm{~s}$ \\
\hline $\mathrm{N}^{+}$ & + & HD & $\rightarrow$ & $\mathrm{NH}^{+}$ & + & $\mathrm{D}$ & $3.2(-10)$ & - & 100 & $\mathrm{~s}$ \\
\hline $\mathrm{C}_{2} \mathrm{H}$ & + & $\mathrm{D}$ & $\rightarrow$ & $\mathrm{C}_{2} \mathrm{D}$ & + & $\mathrm{H}$ & $5.0(-11)$ & 0.5 & 250 & $\mathrm{~s}$ \\
\hline $\mathrm{C}_{2} \mathrm{D}$ & + & $\mathrm{H}$ & $\rightarrow$ & $\mathrm{C}_{2} \mathrm{H}$ & + & $\mathrm{D}$ & $5.0(-11)$ & 0.5 & 832 & $\mathrm{~s}$ \\
\hline $\mathrm{HCN}$ & + & $\mathrm{D}$ & $\rightarrow$ & $\mathrm{DCN}$ & + & $\mathrm{H}$ & $1.0(-10)$ & 0.5 & 500 & s (estimate) \\
\hline $\mathrm{DCN}$ & + & $\mathrm{H}$ & $\rightarrow$ & $\mathrm{HCN}$ & + & $\mathrm{D}$ & $1.0(-10)$ & 0.5 & 500 & s (estimate) \\
\hline $\mathrm{D}^{+}$ & + & $\mathrm{D}^{-}$ & $\rightarrow$ & $\mathrm{D}$ & + & $\mathrm{D}$ & $5.7(-08)$ & -0.5 & - & dl (D-resc) \\
\hline $\mathrm{D}^{+}$ & + & $\mathrm{H}^{-}$ & $\rightarrow$ & $\mathrm{D}$ & + & $\mathrm{H}$ & $4.6(-08)$ & -0.5 & - & $\mathrm{dl}$ (D-resc) \\
\hline $\mathrm{H}^{+}$ & + & $\mathrm{D}^{-}$ & $\rightarrow$ & $\mathrm{D}$ & + & $\mathrm{H}$ & $4.6(-08)$ & -0.5 & - & $\mathrm{dl}$ (D-resc) \\
\hline $\mathrm{D}$ & + & $\mathrm{H}^{-}$ & $\rightarrow$ & $\mathrm{H}$ & + & $\mathrm{D}^{-}$ & $6.4(-09)$ & 0.41 & - & dmd (D-resc) \\
\hline $\mathrm{H}$ & + & $\mathrm{D}^{-}$ & $\rightarrow$ & $\mathrm{D}$ & + & $\mathrm{H}^{-}$ & $6.4(-09)$ & 0.41 & - & dmd (D-resc) \\
\hline $\mathrm{H}_{2}^{+}$ & + & $\mathrm{D}$ & $\rightarrow$ & $\mathrm{HD}^{+}$ & + & $\mathrm{H}$ & $1.07(-09)$ & 0.06 & $41400^{*}$ & $\mathrm{ljb}$ \\
\hline $\mathrm{H}_{2}^{+}$ & + & $\mathrm{D}$ & $\rightarrow$ & $\mathrm{H}_{2}$ & + & $\mathrm{D}^{+}$ & $6.4(-10)$ & - & - & kah \\
\hline $\mathrm{HD}^{+}$ & + & $\mathrm{H}$ & $\rightarrow$ & $\mathrm{H}_{2}^{+}$ & + & $\mathrm{D}$ & $1.0(-09)$ & - & 154 & dmd (D-resc) \\
\hline $\mathrm{HD}^{+}$ & + & $\mathrm{e}^{-}$ & $\rightarrow$ & $\mathrm{H}^{2}$ & + & $\mathrm{D}$ & $3.4(-09)$ & -0.4 & - & str \\
\hline $\mathrm{HD}^{+}$ & + & $\mathrm{H}$ & $\rightarrow$ & HD & + & $\mathrm{H}^{+}$ & $6.4(-10)$ & - & - & $\mathrm{kah}$ \\
\hline $\mathrm{HD}^{+}$ & + & $\mathrm{H}_{2}$ & $\rightarrow$ & $\mathrm{H}_{3}^{+}$ & + & $\mathrm{D}$ & $1.05(-09)$ & - & - & pdf \\
\hline $\mathrm{H}$ & + & $\mathrm{D}^{+}$ & $\rightarrow$ & $\mathrm{HD}^{+}$ & + & $\mathrm{h} \nu$ & $3.9(-19)$ & 1.8 & -20 & $\mathrm{rp}(\mathrm{D}-\mathrm{resc})+\mathrm{fp}$ \\
\hline $\mathrm{D}$ & + & $\mathrm{H}^{+}$ & $\rightarrow$ & $\mathrm{HD}^{+}$ & + & $\mathrm{h} \nu$ & $3.9(-19)$ & 1.8 & -20 & $\mathrm{rp}(\mathrm{D}-\mathrm{resc})+\mathrm{fp}$ \\
\hline $\mathrm{H}_{2}$ & + & $\mathrm{D}$ & $\rightarrow$ & $\mathrm{HD}$ & + & $\mathrm{H}$ & $7.5(-11)$ & - & 3820 & zm (fitted) \\
\hline HD & + & $\mathrm{H}$ & $\rightarrow$ & $\mathrm{H}_{2}$ & + & $\mathrm{D}$ & $7.5(-11)$ & - & 4240 & zm (fitted) \\
\hline $\mathrm{H}_{2}$ & + & $\mathrm{D}^{+}$ & $\rightarrow$ & $\mathrm{H}_{2} \mathrm{D}^{+}$ & + & $\mathrm{h} \nu$ & $1.0(-20)$ & - & - & dmd (D-resc) \\
\hline HD & + & $\mathrm{H}^{+}$ & $\rightarrow$ & $\mathrm{H}_{2} \mathrm{D}^{+}$ & + & $\mathrm{h} \nu$ & $1.0(-20)$ & - & - & dmd (D-resc) \\
\hline $\mathrm{H}_{2}^{+}$ & + & $\mathrm{D}$ & $\rightarrow$ & $\mathrm{H}_{2} \mathrm{D}^{+}$ & + & $\mathrm{h} \nu$ & $7.0(-18)$ & 1.8 & -20 & dmd (D-resc) \\
\hline $\mathrm{HD}^{+}$ & + & $\mathrm{H}$ & $\rightarrow$ & $\mathrm{H}_{2} \mathrm{D}^{+}$ & + & $\mathrm{h} \nu$ & $1.2(-17)$ & 1.8 & -20 & dmd (D-resc) \\
\hline $\mathrm{H}_{2} \mathrm{D}^{+}$ & + & $\mathrm{e}^{-}$ & $\rightarrow$ & $\mathrm{H} \quad+$ & $\mathrm{H}$ & $+\mathrm{D}$ & $4.38(-08)$ & -0.5 & - & $\operatorname{lm}$ \\
\hline $\mathrm{H}_{2} \mathrm{D}^{+}$ & + & $\mathrm{e}^{-}$ & $\rightarrow$ & $\mathrm{H}_{2}$ & + & $\mathrm{D}$ & $4.2(-09)$ & -0.5 & - & $\operatorname{lm}$ \\
\hline $\mathrm{H}_{2} \mathrm{D}^{+}$ & + & $\mathrm{e}^{-}$ & $\rightarrow$ & $\mathrm{H}$ & + & $\mathrm{HD}$ & $1.2(-08)$ & -0.5 & - & $\operatorname{lm}$ \\
\hline $\mathrm{HD}^{+}$ & + & $\mathrm{H}_{2}$ & $\rightarrow$ & $\mathrm{H}_{2} \mathrm{D}^{+}$ & + & $\mathrm{H}$ & $1.05(-09)$ & - & - & pdf \\
\hline $\mathrm{HD}$ & + & $\mathrm{H}_{2}^{+}$ & $\rightarrow$ & $\mathrm{H}_{2} \mathrm{D}^{+}$ & + & $\mathrm{H}$ & $1.05(-09)$ & - & - & dmd (D-resc) \\
\hline HD & + & $\mathrm{H}_{2}^{+}$ & $\rightarrow$ & $\mathrm{H}_{3}^{+}$ & + & $\mathrm{D}$ & $1.05(-09)$ & - & - & dmd (D-resc) \\
\hline
\end{tabular}


Table 7. List of the species for which photo processes can be derived using known cross sections. The photo processes are calculated only from the ground state

\begin{tabular}{|c|c|c|c|}
\hline Species & Processes & Products & References \\
\hline $\mathrm{He}$ & ionisation & $\mathrm{He}^{+}+\mathrm{e}^{-}$ & Band et al. 1990 \\
\hline $\mathrm{C}$ & ionisation & $\mathrm{C}^{+}+\mathrm{e}^{-}$ & Cantù et al. 1981, Hofmann et al. 1983 \\
\hline $\mathrm{C}^{+}$ & ionisation & $\mathrm{C}^{++}+\mathrm{e}^{-}$ & Henry 1970 \\
\hline $\mathrm{C}^{++}$ & ionisation & $\mathrm{C}^{3+}+\mathrm{e}^{-}$ & Osterbrock 1974 \\
\hline $\mathrm{N}$ & ionisation & $\mathrm{N}^{+}+\mathrm{e}^{-}$ & Henry 1970 \\
\hline $\mathrm{O}$ & ionisation & $\mathrm{O}^{+}+\mathrm{e}^{-}$ & Taylor \& Burke 1976, Henry 1970 \\
\hline $\mathrm{Ne}$ & ionisation & $\mathrm{Ne}^{+}+\mathrm{e}^{-}$ & Henry 1970 \\
\hline $\mathrm{Si}$ & ionisation & $\mathrm{Si}^{+}+\mathrm{e}^{-}$ & Chapman \& Henry 1972 \\
\hline \multirow[t]{2}{*}{$\mathrm{C}_{2}$} & ionisation & $\mathrm{C}_{2}^{+}+\mathrm{e}^{-}$ & Padial et al. 1985 \\
\hline & dissociation & $\mathrm{C}+\mathrm{C}$ & Pouilly et al. 1983 \\
\hline \multirow[t]{2}{*}{$\mathrm{CO}$} & ionisation & $\mathrm{CO}^{+}+\mathrm{e}^{-}$ & Hudson 1971 \\
\hline & dissociation & $\mathrm{C}+\mathrm{O}$ & Letzelter et al. 1987 \\
\hline \multirow[t]{4}{*}{$\mathrm{CO}_{2}$} & ionisation & $\mathrm{CO}_{2}^{+}+\mathrm{e}^{-}$ & Hudson 1971, Hitchcock et al. 1980 \\
\hline & fragmentation & $\mathrm{CO}+\mathrm{O}^{+}+\mathrm{e}^{-}$ & Hitchcock et al. 1980 \\
\hline & & $\mathrm{O}+\mathrm{CO}^{+}+\mathrm{e}^{-}$ & Hitchcock et al. 1980 \\
\hline & & $\mathrm{O}_{2}+\mathrm{C}^{+}+\mathrm{e}^{-}$ & Hitchcock et al. 1980 \\
\hline \multirow[t]{2}{*}{$\mathrm{O}_{2}$} & ionisation & $\mathrm{O}_{2}^{+}+\mathrm{e}^{-}$ & $\begin{array}{l}\text { Brion \& Tan 1979, Ogawa \& Ogawa } 1975, \\
\text { Clarke \& Wayne } 1970\end{array}$ \\
\hline & fragmentation & $\mathrm{O}+\mathrm{O}^{+}+\mathrm{e}^{-}$ & Brion \& Tan 1979 \\
\hline \multirow[t]{5}{*}{$\mathrm{N}_{2} \mathrm{O}$} & ionisation & $\mathrm{N}_{2} \mathrm{O}^{+}+\mathrm{e}^{-}$ & Hitchcock et al. 1980 \\
\hline & fragmentation & $\mathrm{NO}^{+}+\mathrm{N}+\mathrm{e}^{-}$ & Hitchcock et al. 1980 \\
\hline & fragmentation & $\mathrm{NO}+\mathrm{N}^{+}+\mathrm{e}^{-}$ & Hitchcock et al. 1980 \\
\hline & fragmentation & $\mathrm{N}_{2}^{+}+\mathrm{O}+\mathrm{e}^{-}$ & Hitchcock et al. 1980 \\
\hline & fragmentation & $\mathrm{N}_{2}^{-}+\mathrm{O}^{+}+\mathrm{e}^{-}$ & Hitchcock et al. 1980 \\
\hline
\end{tabular}

the interaction of light ions with neutrals possessing a permanent electric dipole moment in excess of about 1 Debye. Table 3 gives electric dipole moments for the neutral molecules contained in this database.

\section{Database java applet}

The database java applet can be found on the Rate99 web site at the following URL: http://www.rate99.co.uk. The user can perform searches for reactions which

- include a certain species, either as reactant, product or both;

- are valid at a certain temperature;

- were sourced from a particular reference;

- contain a certain element;

$\ldots$ and so on.

The searches can be made either on the whole database, or on the current results.

Information on an individual reaction can be displayed by selecting that reaction from the results list. The information displayed is an expansion of the flag field (see Sect. 3.1), and includes the formula for calculating the rate coefficient of the reaction, the reference from which the data are sourced and the temperature range over which the data are valid. At this point a graph can also be displayed showing the variation of the reaction rate with temperature, or in the case of interstellar photoreactions (PHOTON) or photoreactions induced by cosmic-rays (CRPHOT), the variation with visual extinction or grain albedo respectively.

A major function of the applet allows the user to build a ratefile interactively by choosing which elements and/or species it is to be composed from and optionally which temperature it is to be used for.

The current ratefile or selection of reactions can be saved at any time onto the user's local machine for use with an equation writer (the format of this file is described in Sect. 3.1). Normally, java applets are denied access to the local filesystem for security reasons. When the applet wants access to the filesystem, the user is alerted and can either grant or deny this permission. For this to work, the browser must support Java 1.1 or later. The implementations of security are (naturally) different between Netscape and Internet Explorer, so to begin with the applet only works with Netscape Navigator v4.5 or above. All this is explained in further detail on the web page itself.

Acknowledgements. We are grateful to all the people who made constructive suggestions and generously provided us with new data sources, particularly Dr. V.G. Anicich who provided an electronic version of his reference list which has been incorporated into the web version of the database, and Dr. J.H. Black whose detailed referee's report enabled us to make significant 
improvements to the database. Astrophysics at UMIST and the work of YHLT in particular are supported via a grant from the Particle Physics and Astronomy Research Council (PPARC). AJM thanks PPARC for a studentship.

\section{References}

Adams N.G., MacIntosh B.J., Smith D., 1990, A\&A 232, 443 Adams N.G., Smith D., Millar T.J., 1984, MNRAS 211, 857

Adams N.G., Smith D., 1985, ApJ Lett. 294, 63

Afeefy H.Y., Liebman J.F., Stein S.E., 2000, "Neutral Thermochemical Data" in NIST Chemistry WebBook, NIST Standard Reference Database Number 69, Mallard W.G. \& Linstrom P.J. (eds.), February 2000, National Institute of Standards and Technology, Gaithersburg, MD 20899 (http://webbook.nist.gov)

Alge E., Adams N.G., Smith D., 1983, J. Phys. B 16, 1433

Andreazza C.M., Singh P.D., Sanzovo G.C., 1995, ApJ 451, 889

Andreazza C.M., Singh P.D., 1997, MNRAS 287, 287

Anicich V.G., Huntress W.T. Jr., 1986, ApJS 62, 553

Anicich V.G., 1993, ApJS 84, 215

Band I.M., Trzhaskovskaya M.B., Verner D.A., Yakovlev D.G., 1990, A\&A 237, 267

Barlow S.G., 1984, Ph.D. Thesis, University of Colorado

Baulch D.C., et al., 1992, J. Phys. Chem. Ref. Data 21, 411

Becker R.S., Hong J.H., 1983, J. Phys. Chem. 87, 163

Bettens R.P.A., Lee H.-H., Herbst E., 1995, ApJ 443, 664

Bohme D.K., 1990, Int. J. Mass Spectrom. Ion Proc. 100, 719

Bohme D.K., Wlodek S., Raksit A.B., 1987, Can. J. Chem. 65, 1563

Botschwina P., Seeger S., Horn M., et al., 1994, AIP Conf. Proc. 312, 321

Botschwina P., 1996, Chem. Phys. Lett. 259, 527

Botschwina P., Horn, M., 1997, J. Mol. Struct. 185, 191

Brion C.E., Tan K.H., 1979, J. Elect. Spectros. Rel. Phen. 17, 101

Brownsword R.A., et al., 1997, ApJ 485, 195

Cantù A.M., Mazzoni M., Pettini M., Tozzi G.P., 1981, Phys. Rev. A 23, 1223

Chapman R.D., Henry R.J.W., 1972, ApJ 173, 243

Clark I.D., Wayne R.P., 1970, Mol. Phys. 18, 523

Clary D.C., Haider N., Husain D., Kabar M., 1994, ApJ 422, 416

Copp N.W., et al., 1982, Chem. Phys. Lett. 88, 508

Crosswell K., Dalgarno A., 1985, ApJ 289, 618

Dalgarno A., Du M.L., You J.H., 1990, ApJ 349, 675

Dalgarno A., Lepp S., 1987, in Astrochemistry, Tarafdar S.P., Varshni M.P. (eds.). Dordrecht: Reidel, p. 109

Dalgarno A., McDowell M.R.C., 1956, Proc. Phys. Soc. London A 69,615

Decker B.K., Adams N.G., 1997, Int. J. Mass Spectr. Ion Proc. $165 / 166,257$

Dheandanoo S., Forte L., Fox A., Bohme D.K., 1986, Can. J. Chem. 64, 641

Donnelly V.M., Pasternack L., 1979, Chem. Phys. 39, 427

Draine B.T., 1978, ApJS 36, 595

Federer W., Villinger H., Lindinger W., Richter R., Ferguson E.E., 1986, Chem. Phys. Lett. 123, 12

Field D., Adams N.G., Smith D., 1980, MNRAS 192, 1
Filseth S.V., et al., 1979, Chem. Phys. Lett. 61, 288

Forst W., 1991, J. Phys. Chem. 95, 3612

Frommhold L., Pickett H.M., 1978, Chem. Phys. 28, 441

Frost M.J., Sharkey P., Smith I.W.M., 1991, J. Chem. Soc. Faraday Trans. 91, 305

Giles K., 1990, Ph.D. Thesis, University of Birmingham

Giles K., Adams N.G., Smith D., 1989, Int. J. Mass Spectrom. Ion Proc. 89, 303

Gerlich D., Horning S., 1992, Chem. Rev. 92, 1509

Gingerich K.A., Finkbeiner H.C., Schmude R.W., 1994, JACS 116,3884

Gredel R., Lepp S., Dalgarno A., 1987, ApJ 323, L137

Gredel R., Lepp S., Dalgarno A., Herbst E., 1989, ApJ 347, 289

Haider N., Husain D., 1993a, J. Photochem. Photobiol. A70, 119

Haider N., Husain D., 1993b, J. Chem. Soc. Farad. Trans. 89, 7

Harding L.B., Guadagnini R., Schatz G.C., 1993, J. Phys. Chem. 97, 5472

Henry R.J.W., 1970, ApJ 161, 1153

Herbst E., 1985, ApJ 291, 226

Herbst E., 1987, ApJ 313, 867

Herbst E., Adams N.G., Smith D., Defrees D.J., 1987, ApJ 312,351

Herbst E., Adams N.G., Smith D., Giles K., 1989, J. Chem. Soc. Farad. Trans. 85, 1655

Herbst E., DeFrees D.J., Koch W., 1989, MNRAS 237, 1057

Herbst E., Giles K., Smith D., 1990, ApJ 358, 468

Herbst E., Lee H.-H., 1997, ApJ 485, 689

Herbst E., Leung C.M., 1986, ApJ 310, 378

Herbst E., Leung C.M., 1989, ApJS 69, 271

Herbst E., Leung C.M., 1990, A\&A 233, 177

Herbst E., Millar T.J., Wlodek S., Bohme D.K., 1989, A\&A 222,205

Herbst E., Terzieva R., Talbi D., 2000, MNRAS 311, 869

Herd C.R., Adams N.G., Smith D., 1990, ApJ 349, 388

Hitchcock A.P., Brion C.E., van der Wiel M.J., 1980, Chem. Phys. 45, 461

Hofmann H., Saha H.P., Trefftz E., 1983, A\&A 126, 415

Hudson R.D., 1971, Rev. Geophys. Spa. Phys. 9, 305

Husain D., Norris P.E., 1979, Far. Disc. Chem. Soc. 67, 273

Karpas Z., Anicich V.G., Huntress W.T., 1979, J. Chem. Phys. 70, 2877

Karpas Z., Meot-ner M., 1989, J. Phys. Chem. 93, 1859

Kimura M., Dalgarno A., 1993, Chem. Phys. Lett. 211, 454

Larson Å., et al., 1998, ApJ 505, 459

Larsson M., et al., 1996, A\&A 309, L1

Lee H.-H., Bettens R.P.A., Herbst E., 1996, A\&AS 119, 111

Leen T.M., Graff M.M., 1988, ApJ 325, 411

Letzelter C., Eidelsberg M., Rostas F., 1987, Chem. Phys. 114, 273

Leung C.M., Herbst E., Huebner W.F., 1984, ApJS 56, 231

Linder F., Janev R.K., Botero J., 1995, in Atomic and Molecular Processes in Fusion Edge Plasmas, Janev R.K. (ed.). Plenum Press, New York, p. 397

MacGregor M., Berry R.S., 1973, J. Phys. B 6, 181

Mallard W.G., Westley F., Herron J.T., Hampson R.F., Frizzell D.H., 1998, NIST Chemical Kinetics Database, Version 2Q98, National Institute of Standards and Technology, Gaithersburg, MD

Maluendes S., McLean A.D., Herbst E., 1993, ApJ 417, 181 
Mann D.M., 1977, Chem. Phys. Lett. 47, 106

Marston G., Nesbitt F.L., Stief L.J., 1989, J. Chem. Phys. 91, 3483

McEwan M.J., Scott G.B.I., Adams N.G., et al., 1999, ApJ 513,287

Millar T.J., 1991, A\&A 242, 241

Millar T.J., Adams N.G., Smith D., Clary D.C., 1985, MNRAS 216,1025

Millar T.J., Adams N.G., Smith D., Lindinger W., Villinger H., 1986, MNRAS 221, 673

Millar T.J., Bennett A., Herbst E., 1987, MNRAS 229, 41P

Millar T.J., Farquhar P.R.A., Willacy K., 1997, A\&AS 121, 139

Millar T.J., Herbst E., 1990, A\&A 231, 466

Millar T.J., Herbst E., Charnley S.B., 1991, ApJ 369, 147

Millar T.J., Nejad L.A.M., 1985, MNRAS 217, 507

Mitchell G.F., 1984, ApJ 287, 665

Mitchell G.F., Deveau T.J., 1983, ApJ 266, 646

Mitchell J.B.A., 1990, Phys. Rep. 186, 215

Nahar S.N., 1999, ApJS 120, 131

Nahar S.N., Pradhan A.K., 1997, ApJS 111, 339

Nesbitt F.L., Martson G., Stief L.J., 1990, J. Phys. Chem. 94, 4946

Ogawa S., Ogawa M., 1975, Can. J. Phys. 53, 1845

Osterbrock D.E., 1974, Astrophysics of Gaseous Nebulae. W.H. Freeman \& Co., San Francisco

Oswald M., Botschwina P., 1995, J. Mol. Spectros. 169, 181

Padial N.T., Collins L.A., Schneider B.I., 1985, ApJ 298, 369

Pauzat F., Ellinger Y., McLean A.D., 1991, ApJ 369, L13

Petrie S., Freeman C.G., McEwan M.J., 1992, MNRAS 257, 438

Petuchowski S.J., Dwek E., Allen J.E. Jr., Nuth III J.A., 1989, ApJ 342, 406

Pineau des Forêts G., Flower D.R., Hartquist T.W., Dalgarno A., 1986, MNRAS 220, 801

Pineau des Forêts G., Roueff E., Flower D.R., 1990, MNRAS 244,668

Pouilly B., Robbe J.M., Schamps J., Roueff E., 1983, J. Phys. B 16, 437

Prasad S.S., Huntress W.T. Jr., 1980, ApJS 43, 1

Prasad S.S., Huntress W.T. Jr., 1982, ApJ 250, 590

Raksit A.B., Warneck P., 1980, J. Chem. Soc. Farad. II 76, 1084

Ramaker D.E., Peek J.M., 1976, Phys. Rev. A 13, 58

Rawlings J.M.C., 1992 (private communication)

Rawlings J.M.C., Williams D.A., Canto J., 1988, MNRAS 230, 695

Reisler H., et al., 1980, Chem. Phys. 47, 49

Roberge W.G., Jones D., Lepp S., Dalgarno A., 1991, ApJS 77,287
Schilke T., Walmsley C.M., Pineau des Forêts G., Roueff E., Flower D.R., Guilloteau S., 1992, A\&A 256, 595

Sen A., Anicich V.G., Federman S.R., 1992, ApJ 391, 141

Sidhu K.S., Miller S., Tennyson J., 1992, A\&A 255, 453

Sims I.R., Queffelec J.-L., Defrance A., et al., 1994, J. Chem. Phys. 100, 4229

Sims I.R., Queffelec J.-L., Travers D., et al., 1993, Chem. Phys. Lett. 211,461

Smith D., Adams N.G., 1984, ApJ 284, L13

Smith D., Adams N.G., Alge E., 1982a, ApJ 263, 123

Smith D., Adams N.G., Alge E., 1982b, J. Chem. Phys. 77, 1261

Smith D., Adams N.G., Giles K., Herbst E., 1988, A\&A 200, 191

Smith D., MacIntosh B.J., Adams N.G., 1989, J. Chem. Phys. 90, 6213

Smith D., Spanel P., 1993, Chem. Phys. Lett. 211, 454

Smith D., Spanel P., Mayhew C.A., 1992, Int. J. Mass Spectrom. Ion Proc. 117, 457

Smith D., Spanel P., Millar T.J., 1994, MNRAS 266, 31

Smith I.W.M., 1989, ApJ 347, 282

Smith M.A., 1993, J. Chem. Soc. Farad. Trans. 89, 2210

Stancil P.C., Schultz D.R., Kimura M., Gu J.-P., Hirsch G., Buenker R.J., 1999, A\&AS 140, 225

Stief L.J., Marston G., Nava D.F., Payne W.A., Nesbitt F.L., 1988, Chem. Phys. Lett. 147, 570

Strömholm C., et al., 1995, Phys. Rev. A 52, R4320

Suernam R.D., Lovas F.J., 1994, ApJ 429, L89

Suzuki H., Yamamoto S., Ohishi M., et al., 1992, ApJ 392, 551

Talbi D., Ellinger Y., Herbst E., 1996, A\&A 314, 688

Taylor K.T., Burke P.G., 1976, J. Phys. B 9, L353

Thorne L.R., Anicich V.G., Prasad S.S., Huntress W.T. Jr., 1984, ApJ 280, 139

Tsang W., Hampson R.F., 1986, J. Phys. Chem. Ref. Data 15, 1087

van Dishoeck E.F., Black J.H., 1988, ApJ 334, 771

van Dishoeck E.F., 1987, in Astrochemistry, Vardya M.S. and Tarafdar S.P. (eds.). Reidel, Dordrecht, p. 51

Viggiano A.A., Paulson J.F., 1983, J. Chem. Phys. 79, 2241

Vikor L., Al-Khalili A., Danared H., et al., 1999, A\&A 344, 1027

Watson W.D., 1976, Rev. Mod. Phys. 48, 513

Wilson P.F., McEwan M.J., Meot-ner M., 1994, Int. J. Mass Spectrom. Ion Proc. 132, 149

Wlodek S., Bohme D.K., Herbst E., 1988, MNRAS 235, 493

Zhang J.Z.H., Miller W.H., 1989, J. Chem. Phys. 91, 1528

Zygelman B., Dalgarno A., Kimura M., Lane N.F., 1989, Phys. Rev. A 40, 2340 